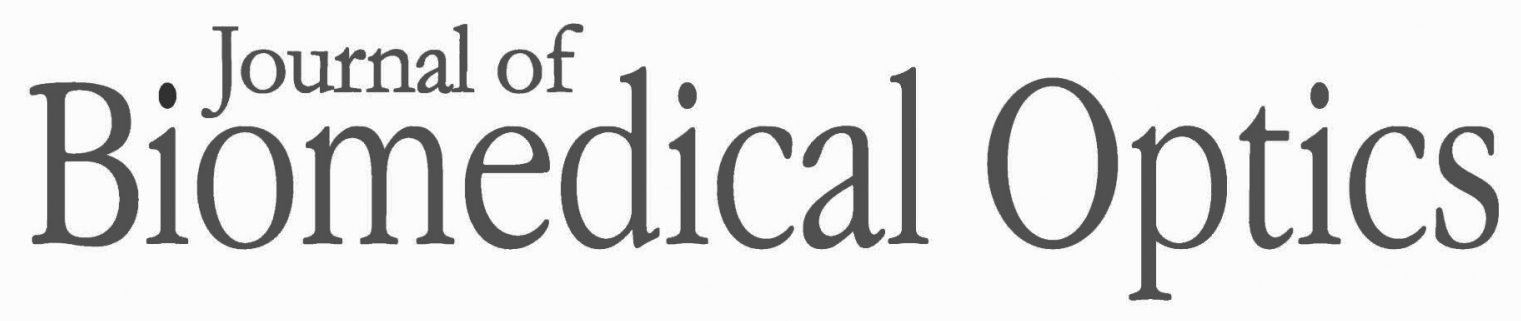

SPIEDigitalLibrary.org/jbo

\title{
Ocular microtremor measurement using laser-speckle metrology
}

Emer Kenny

Davis Coakley

Gerard Boyle

\section{O SPIE}




\title{
Ocular microtremor measurement using laser-speckle metrology
}

\author{
Emer Kenny, ${ }^{a, b}$ Davis Coakley, ${ }^{b}$ and Gerard Boyle ${ }^{c}$ \\ ${ }^{a}$ Trinity College Dublin, School of Medicine, Dublin 2, Ireland \\ bMercer's Institute for Research on Ageing, St. James's Hospital, Dublin 8, Ireland \\ 'St. James's Hospital, Department of Medical Physics and Bioengineering, Dublin 8, Ireland
}

\begin{abstract}
We describe a novel, noninvasive measurement approach for recording a small involuntary tremor of the eye known as ocular microtremor. The method is based on measuring out-of-plane angular displacements of a target by using laser-speckle correlation of images recorded in the Fourier plane of a lens. The system has a dynamic range of 4 to $5000 \mu \mathrm{rad}$, resolution of $4 \mu \mathrm{rad}$, and a bandwidth of $250 \mathrm{~Hz}$. The design and optimization of the system is presented with an in vitro validation of the system against its specification. $\odot$ The Authors. Published by SPIE under a Creative Commons Attribution 3.0 Unported License. Distribution or reproduction of this work in whole or in part requires full attribution of the original publication, including its DOI. [DOI: 10.1117/1.JBO.18.1.016010]
\end{abstract}

Keywords: ocular microtremor; speckle metrology; angular speckle displacement; speckle correlation; subpixel registration; electron multiplying charged couple device.

Paper 12733 received Nov. 12, 2012; revised manuscript received Dec. 12, 2012; accepted for publication Dec. 18, 2012; published online Jan. 11, 2013.

\section{Introduction}

Fixational eye movements are present in all subjects even when the eye appears to be at rest. These fixational movements are composed of three types of involuntary eye movements: microsaccades, drift, and ocular microtremor (OMT). ${ }^{1,2}$

OMT is the smallest of the involuntary eye movements. It has an amplitude range of 150 to $2500 \mathrm{~nm}$ (12 to $216 \mu \mathrm{rad}$ rotational) peak-to-peak, with a typical amplitude of $1 \mu \mathrm{m}$ (87 $\mu \mathrm{rad})$. The frequency range of OMT is between 20 to $150 \mathrm{~Hz}$ with an average peak frequency of $84 \mathrm{~Hz}^{3}$ for a normal subject. It is a nonperiodic, continual, high-frequency, wavelike physiological tremor. ${ }^{4,5}$ Microtremor occurs simultaneously with drift, which is a slow, continuous irregular movement that takes place between the more rapid and intermittent microsaccades.

Clinical use of OMT has been investigated in coma, ${ }^{6} \mathrm{MS},{ }^{7}$ Parkinson's disease, ${ }^{8}$ and depth of anaesthesia. ${ }^{9,10}$ Recent studies have attempted to understand the particular role of OMT in vision. ${ }^{11}$ It is believed OMT arises from brainstem neurons. ${ }^{5,12,13}$

Most measurements of OMT to date have relied on an eyecontacting probe method. ${ }^{14,15}$ Drawbacks of these probes include the requirement to anaesthetise the eye, inter-observer variability, probe influence on the measurement result and patient discomfort. ${ }^{16,17}$

Despite its potential, a full understanding of OMT is incomplete due to the absence of a device to measure it noninvasively. For practical clinical use, a noncontacting method for OMT measurement would be most suitable. Ideally the device would be of a high resolution yet robust enough to measure OMT in a clinical environment. Boyle et al. ${ }^{18}$ proposed a speckle interferometry method for noncontact measurement of OMT from the eye sclera. The system achieved a resolution suitable for OMT measurement, though the setup was difficult to implement due

Address all correspondence to: E. Kenny, Trinity College Dublin, School of Medicine, Dublin 2, Ireland. Tel: +353 1 4284882l; E-mail: kennye3@tcd.ie to the bulk optics utilized. A promising outcome of the work by Boyle was that it showed the sclera to be an appropriate surface for measuring OMT using speckle-based methods. As a continuation of the work by Boyle, Ryle et al. ${ }^{19}$ developed a speckle interferometry OMT measurement system using laser diodes in order to reduce the physical size of the system. However, again the system was difficult to implement.

Here we investigate the feasibility of using laser-speckle correlation to track OMT from the surface of the sclera and outline the requirements for such a measurement system. Specifically, we investigate the application of a laser-speckle technique using Fourier plane imaging to measure OMT. The technique potentially presents advantages over other optical eye-movement measurement techniques in terms of resolution, ease of setup, and robustness to misalignment. To our knowledge this approach has not been applied previously to eyemovement measurement and would represent an important addition to the relatively limited number of noncontact eye movement techniques available, ${ }^{20,21}$ if its utility can be demonstrated. While the basic optical technique has been described elsewhere, it has been validated and applied only for the measurement of relatively simple displacements of inanimate objects, such as small rotation of metal shafts. The eye presents a much more challenging target. To adapt and validate the technique for OMT measurement requires the issues of eye safety, background eye movement, and biospeckle to be resolved. The former two issues are addressed in this paper, as moving to investigate biospeckle effects in vivo initially requires the basic technique to be proven in vitro at eye safe power levels against the known motion of a target. To address eye safety, we implement the technique here at much lower laser power levels than have been used previously and utilize a high-sensitivity electron multiplying charged couple device camera to attempt to compensate for the consequent drop in signal and potential loss of resolution. OMT takes place against a background of "contaminating" background eye and head movements. A downside of the fundamental optical technique used here is that it is vulnerable to 
failure through decorrelation effects for "large" movements. A series of validation tests are presented here to realistically challenge the proposed measurement approach and to determine if it can be configured to resolve the very low-amplitude rotations caused by OMT, despite the necessary restrictions on laser power and the presence of larger amplitude background motion.

\subsection{Measurement Requirements}

The reported amplitude of OMT varies in the literature. For metrology purposes, most OMT measurements that have been recorded using the contacting probes method give the amplitude in terms of nanometers. For our purposes, we convert these figures to units of angular rotation of the eye by noting that $87 \mu \mathrm{rad}$ is equivalent to a $1 \mu \mathrm{m}$ displacement for a typical eye diameter of $23 \mathrm{~mm}$

\subsubsection{Frame rate}

The frame rate of a camera attempting to capture speckle moving at OMT frequencies must obey the Nyquist sampling theorem. To do this, the sampling frequency $\left(f_{s}\right)$ must be greater than or equal to twice the highest frequency contained in the signal, i.e., for OMT measurement the frame rate must be $\geq 2(150 \mathrm{~Hz})$. In the case of living tissue such as the eye, biospeckle will be present in the signal captured by the camera. At present, the rate of biospeckle motion in the eye is unknown, and so a higher sampling frequency than that required for OMT alone would be beneficial. A frame rate of $500 \mathrm{~Hz}$ (500 frames per second) is high enough to obey the Nyquist theorem and avoid aliasing of the OMT signal.

\subsubsection{Dynamic range and resolution}

Generally a dynamic range of 2 to $216 \mu \mathrm{rad}(25$ to $2500 \mathrm{~nm}$ peak-to-peak) and system resolution of $2 \mu \mathrm{rad}$ has been found to be adequate to track the OMT signal. ${ }^{15,16,18}$ Although this dynamic range is sufficient to capture OMT, when a large intermittent microsaccade or other movement occurs, a momentary saturation arises in the measurement. More recent studies have used a dynamic range of $2 \mu \mathrm{rad}$ to $9.52 \mathrm{mrad}(25 \text { to } 110 \mu \mathrm{m})^{11,22}$ so that other fixational movements could also be recorded. In a contacting probe measurement system, the system dynamic range and resolution are critical parameters.

In a speckle correlation system, dynamic range will be determined by the maximum allowable frame displacement relative to a reference frame taken at the start of the measurement. A large frame-to-frame displacement will cause a decorrelation in the measurement and reduce the accuracy. Some intermittent resetting of the reference frame could be tolerated in response to large movements. This would be apparent as a discontinuity in the measured movement trace. However, at a minimum, the system needs to tolerate the expected frame-to-frame displacement. Therefore some a priori knowledge of estimates for how large these displacements might be is needed in order to design a system capable of measuring OMT against a background of other movements.

\subsubsection{Influence of other eye movements on measurement}

Other eye movements can lead to decorrelation of the speckle correlation images. Table 1 displays the amplitude, frequency, and velocity ranges estimated from the literature for each of the fixational eye movements. Drift and microsaccades have
Table 1 Characteristics of the fixational eye movements. Velocity measurements are based on the amplitude and frequency (unless referenced).

\begin{tabular}{lcccc}
\hline & $\begin{array}{c}\text { Amplitude } \\
(\mu \mathrm{rad})\end{array}$ & $\begin{array}{c}\text { Frequency } \\
(\mathrm{Hz})\end{array}$ & $\begin{array}{c}\text { Maximum } \\
\text { velocity } \\
\text { (mrad/s) }\end{array}$ & $\begin{array}{c}\text { Maximum } \\
\text { rotation } \\
\text { per frame at } \\
500 \mathrm{FPS} \\
(\mu \mathrm{rad} / \mathrm{frame})\end{array}$ \\
\hline OMT & $\begin{array}{c}12 \text { to } 200 \\
\text { peak to peak }\end{array}$ & $\sim 80$ & 50 & 101 \\
Drift & 290 & 2 to 5 & $9^{2}$ & 18 \\
Microsaccade & 1450 & 1 to 2 & $637^{2}$ & 1274 \\
\hline
\end{tabular}

an amplitude of about $290 \mu \mathrm{rad}$ and $1.45 \mathrm{mrad}$, respectively. Drift has a frequency of 2 to $5 \mathrm{~Hz}$ and, as previously mentioned, occurs simultaneously with OMT. Microsaccades are more rapid and sporadic and occur approximately twice every second. An imaging system recording speckle from the eye would capture the net displacement of the eye surface resulting from all three fixational movements. Post processing of the images must therefore include a filtering method to separate the OMT signal from the other eye movements.

\subsubsection{Influence of body movements on measurement}

Unwanted head movements can occur during eye-movement measurement. These can have a negative influence on the accuracy of measurements since the eye globes participate in the compound movements of the head. Natural functions such as breathing and heart rate can affect these head movements. Head movements vary in amplitude between measurements and between subjects, and so it is difficult to establish their amplitude. Table 2 displays the amplitude, frequency, and velocity ranges estimated from the literature for each of the unwanted head movements. To stabilize the head while recordings are being taken, a headrest may be used. It has been shown, however, that standard ophthalmic headrests do not eliminate all head movement and amplitudes in the range of $\pm 9 \mathrm{mrad}$ $( \pm 100 \mu \mathrm{m})$ at 2 to $3 \mathrm{~Hz}$ are still possible with their use. ${ }^{23}$

In a clinical environment, a portable handheld measurement device would be the ideal solution in cases where the patient is not mobile and cannot place his own head in a headrest. Shaking of the hands while holding the device would add additional noise to the system. The outstretched arm has a natural tremor

Table 2 Characteristics of other body movements that may influence OMT. Velocity measurements are calculated based on the amplitude and frequency.

\begin{tabular}{lcccc} 
& $\begin{array}{c}\text { Amplitude } \\
\text { (mrad) }\end{array}$ & $\begin{array}{c}\text { Frequency } \\
\text { (Hz) }\end{array}$ & $\begin{array}{c}\text { Maximum } \\
\text { velocity } \\
\text { (mrad/s) }\end{array}$ & $\begin{array}{c}\text { Maximum } \\
\text { rotation } \\
\text { per frame at } \\
500 \text { FPS } \\
\text { ( } \mathrm{rrad} / \text { frame) }\end{array}$ \\
\hline Headrest & $9^{23}$ & 2 to 3 & 85 & 170 \\
Hand & 4 to $17^{16,24}$ & 7 to 12 & 641 & 1282 \\
\hline
\end{tabular}


of 4 to $17 \mathrm{mrad}$ (50 to $200 \mu \mathrm{m}$ ) in amplitude with a frequency of 7 to $12 \mathrm{~Hz}^{16,24}$

\subsubsection{Required frame-to-frame measurement ability}

In order to determine the required frame-to-frame measurement ability of the system, the likely displacement per frame of all movements that may influence the OMT signal must be calculated. It is clear that in a headrest device, the dominant components are likely to be microsaccades. Treating the above velocities as vectors, the maximum possible angular velocity will occur for a situation where all velocities are in the same direction. In this case, the maximum velocity would be

$$
\begin{aligned}
& (\text { OMT }+ \text { drift }+ \text { microsaccades }+ \text { head }) \\
& \quad=(50+9+637+85) \mathrm{mrad}=781 \mathrm{mrad} / \mathrm{s} .
\end{aligned}
$$

At our proposed frame rate of 500FPS, the maximum possible displacement per frame is estimated to be $1.56 \mathrm{mrad}$.

Including motion from a handheld device the maximum velocity would increase to $1422 \mathrm{mrad} / \mathrm{s}$ and the maximum possible displacement per frame would increase to $2.84 \mathrm{mrad}$. From these figures, it is apparent that hand movement would become the dominant motion in a measurement system. The velocity and displacement of the total movements almost double when hand movement is included. At present, the measurement device is table mounted and so further calculations will be derived for a non-handheld device.

The unwanted eye, head, and hand movements have frequencies below that of OMT, and so a filter may be implemented to remove them with signal processing. The filtering process would not work however if the frame-to-frame decorrelation caused by the head and hand movements is large. The effects of this possible decorrelation must therefore be investigated and quantified.

In summary, the following parameters are required for an OMT speckle correlation measurement system: (1) a resolution of $2 \mu \mathrm{rad}$; (2) a dynamic range of $2 \mu$ to $9.52 \mathrm{mrad}$; (3) a frame rate of 500FPS; and (4) a minimum frame-to-frame measurement ability of $1.56 \mathrm{mrad}$.

\subsection{Fourier Plane Measurement Technique}

Figure 1 shows the arrangement used by Rose et al. ${ }^{25,26}$ for angular speckle displacement measurement. Light from a collimated laser beam is directed through a beam splitter to a target where it is then reflected back to an image sensor placed in the Fourier plane (i.e., $z_{2}=f$ ) of a lens with aperture $\sigma$. The out-ofplane angular rotation $(\theta)$ of the object is transformed to a linear displacement $\left(p_{x}\right)$ in the Fourier plane of a lens. An image sensor placed in the Fourier plane records the speckle patterns over a certain time. The speckle images are then post-processed using digital-image correlation to measure the linear displacement between speckle images. This linear displacement is then converted to angular rotation of the object using the simple equation $^{25}$

$$
\theta=\frac{p_{x}}{2 f}
$$

Speckle techniques recorded in the imaging plane are dependent on a number of variables such as the lens focal length, working distance, numerical aperture, the wavelength of the

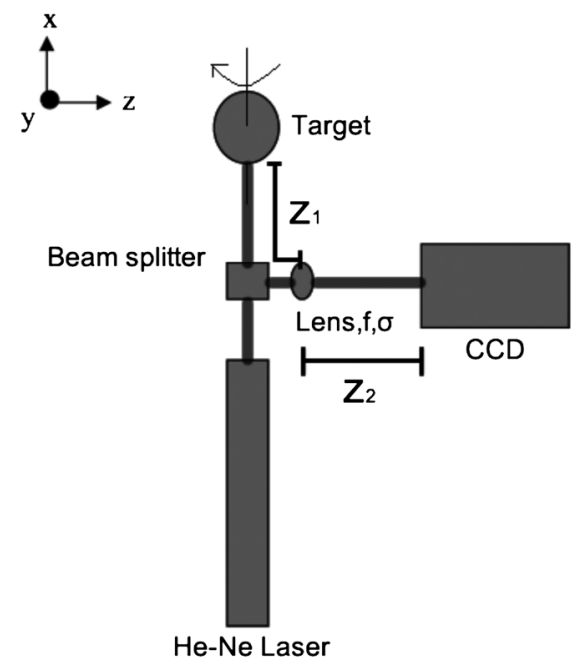

Fig. 1 Setup for Fourier plane measurement technique.

light, and the target shape. An advantage of the Fourier technique implemented by Rose et $\mathrm{al}^{26}$ is that the angular displacement is dependant only on the linear speckle image displacement and on the lens focal length $(f)$, hence the setup of the system is much less stringent than that of an imaging plane technique.

Other advantages in terms of precise OMT measurement with this technique are the proven range and sensitivity that can be achieved. In their paper, Rose et al. ${ }^{26}$ report a resolution of $5 \mu \mathrm{rad}$ for the technique; this is close to the ideal OMT system resolution of $2 \mu \mathrm{rad}$. Further, the method has been proven to be insensitive to target shape and to target distance $\left(z_{1}\right)$. The latter is important to OMT measurement, since it would allow for simple setup of the equipment without a rigid working distance. A fundamental advantage of the technique designed by Rose et al. ${ }^{26}$ is that their system is sensitive only to rotation. This is significant for OMT measurement as it would imply that the method is robust and insensitive to any left/right or back/front motion of the subject as the measurement is being taken.

\subsection{Design Parameters}

Using the Rose method, as described above, there are only three variables in the setup: the beam radius $r_{s}$, lens focal length $f$, and lens aperture $\sigma$. In the following section, the influence of these parameters on the design of the system will be discussed with an aim to find optimized values for an OMT measurement system and to calculate the theoretical dynamic range of our system. Laser power constraints due to eye safety are also discussed.

Due to the low light levels in this experiment, it would be prudent to use a smaller focal length lens in order to keep the target-to-lens-to-camera-sensor distance shorter and hence collect more reflected light at the sensor. An $f=150 \mathrm{~mm}$ lens was considered to be a good compromise between achievable resolution and reflected intensity, reducing the estimated resolution to $4 \mu \mathrm{rad}$.

To achieve a speckle size that is at least as large as one pixel, using a focal length of $f=150 \mathrm{~mm}$ as derived above, the beam radius must not be larger than $1.26 \mathrm{~mm}$ for a $632.8-\mathrm{nm}$ laser. 
Using the lateral sclera as a target, the beam diameter must be able to fit within the accessible space on the sclera. For a typical adult eye, the available space is approximately $4 \mathrm{~mm}$. Therefore a beam radius of up to $2 \mathrm{~mm}$ would fit on the sclera.

A small beam radius is desirable because it will provide a higher signal-to-noise ratio and a higher speckle size. For this reason, we opted not to adjust the actual beam radius of the laser used in this experiment and the given radius was $0.6 \mathrm{~mm}$. This beam radius is well within the available space on the sclera.

Using the theory outlined in Rose et al. ${ }^{25,26}$ and assuming fully developed speckle, to achieve a decorrelation factor of 0.9 or higher for a maximum possible angular displacement of $\theta=1.56 \mathrm{mrad}$ (as derived in Sec. 1.1) with a lens focal length of $f=150 \mathrm{~mm}$, then the lens aperture must be greater than or equal to $2.04 \mathrm{~mm}$. For our setup, we chose a standard optic size of $25 \mathrm{~mm}$.

Large movements will decorrelate images from frame-toframe. This will have an impact on the dynamic range. The ceiling on the dynamic range achievable by the system can be determined using Eq. (1). From this, the angular displacement required to translate a single speckle across the image sensor can be calculated. For a lens focal length of $150 \mathrm{~mm}$ and a $128 \times 128$ image sensor with a $24 \mu \mathrm{m}$ pixel pitch the angular displacement required to displace one speckle fully across the sensor is $10 \mathrm{mrad}$. Therefore, in a scenario where each frame is correlated with an original undisplaced reference frame, the theoretical maximum achievable dynamic range for our system is $10 \mathrm{mrad}$.

The laser power used in an OMT measurement system must be restricted to a safe level and yet be high enough to achieve a reasonable signal-to-noise ratio at the imaging detector. Ideally the laser light would be visible so that alignment of the laser with the eye sclera is easily achievable. In this study, a visible red helium neon $(632.8 \mathrm{~nm} \mathrm{HeNe})$ laser was chosen for the OMT tracking device.

Using International Electrotechnical Commission (IEC 60825-1 $)^{27}$ and American National Standards Institution (ANSI Z136.1), ${ }^{28,29}$ limits for the safe exposure of eyes and skin to laser radiation the MPE values for a direct intrabeam viewing of a 632.8-nm wavelength laser for 10s exposure time to an eye with a 7-mm pupil diameter was found to be $388.65 \mu \mathrm{W}$. It should be noted that this value is for an exposure to the retina, however, in normal use we are aiming the laser at the sclera and hence there is less risk of retinal eye damage.

To summarize, the following design parameters were calculated for our system: (1) a lens focal length of $150 \mathrm{~mm}$; (2) a beam radius of $0.6 \mathrm{~mm}$; (3) an aperture size of $25 \mathrm{~mm}$; (4) a laser wavelength of $632.8 \mathrm{~nm}$; and (5) a laser power of $136 \mu \mathrm{W}$.

\section{Methods}

In the following experiments, we aim to demonstrate the feasibility of using the speckle correlation method for measuring the rotation of objects with OMT-like movements. The proposed measurement system must be tested at eye-safe laser levels for its ability to meet OMT measurement specifications in terms of accuracy, resolution, range, and robustness to larger eye movements. To perform these tests both an OMT simulator and a manually adjusted gimbal were used to simulate the moving eye. A piece of cardboard was inserted into the gimbal to act as the target material and, similarly, another piece of the same cardboard was attached to the plastic of the simulator.

The optical setup for the experiment is shown in Fig. 2, the EMCCD sensor is placed in the fourier plane of the lens, i.e., at

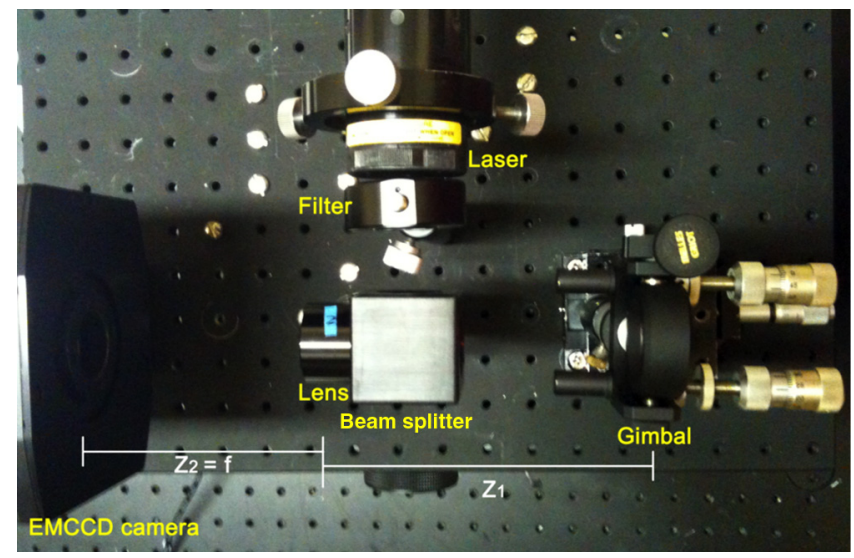

Fig. 2 Experimental setup.

$z_{2}=f$. The target is illuminated by a $632.8 \mathrm{~nm}$ plane polarized $\mathrm{HeNe}$ laser, which is reduced down to an eye-safe power of $139 \mu \mathrm{W}$ by the use of a neutral density filter and a beamsplitter. The speckle pattern generated on the target is collected by an EMCCD camera (Cascade $128+$, Roper Scientific ${ }^{30}$ ) operating at $500 \mathrm{~Hz}$ with a $128 \times 128$ array of $24 \mu \mathrm{m}$ pixels, and a $100 \%$ fill factor. A 5 second (2500 frames) reading is taken for each measurement. The 8-bit digitized speckle images are then sent to the numerical software package Matlab ${ }^{31}$ for processing. The analysis procedure is shown in Fig. 3.

During the processing each displaced speckle-image frame is cross-correlated with the original reference speckle-image frame. The full $128 \times 128$ pixels are used for every image during the cross-correlation analysis. To increase the accuracy in finding the exact correlation peak, and consequently the speckle displacement, the location of the cross-correlation peak is calculated with a subpixel algorithm. The subpixel correlation method used in this experiment is based on the curve-fitting method as described by Hung et al. ${ }^{32}$ The change in correlation peak location over time is used to calculate the pixel displacement of the speckle images.

Using Eq. (1), the calculated pixel displacement, $p_{x}$, is converted to the measured angular displacement, $\theta$. To remove noise outside the desired frequencies, the signal is then filtered using a digital Butterworth filter of order five with a bandwidth of 20 to $150 \mathrm{~Hz}$. A peak detection algorithm is used to measure each peak and trough in the recovered signal. To find the amplitude of the signal, each trough is subtracted from each peak, and the mean value is taken to be the peak-to-peak amplitude of the measured signal. To calculate the frequency content of the signal, a periodogram is utilised to estimate the power spectral density.

\subsection{Simulator Tests}

An OMT simulator was used to test the measurement resolution, amplitude response, and frequency response of the system. The simulator replicates OMT movement in terms of typical frequencies and amplitudes. It was first designed by Sheahan ${ }^{16}$ but has since been revised and updated. A picture of the simulator is shown in Fig. 4. The two outer piezoelectric bimorphs of the simulator displace in response to an applied voltage, and the inner bimorph is used as a reference for calibration. The bimorphs are attached to a piece of plastic on one end. As the bimorphs bend the attached plastic tilts at an angle that is proportional to the applied voltage. 


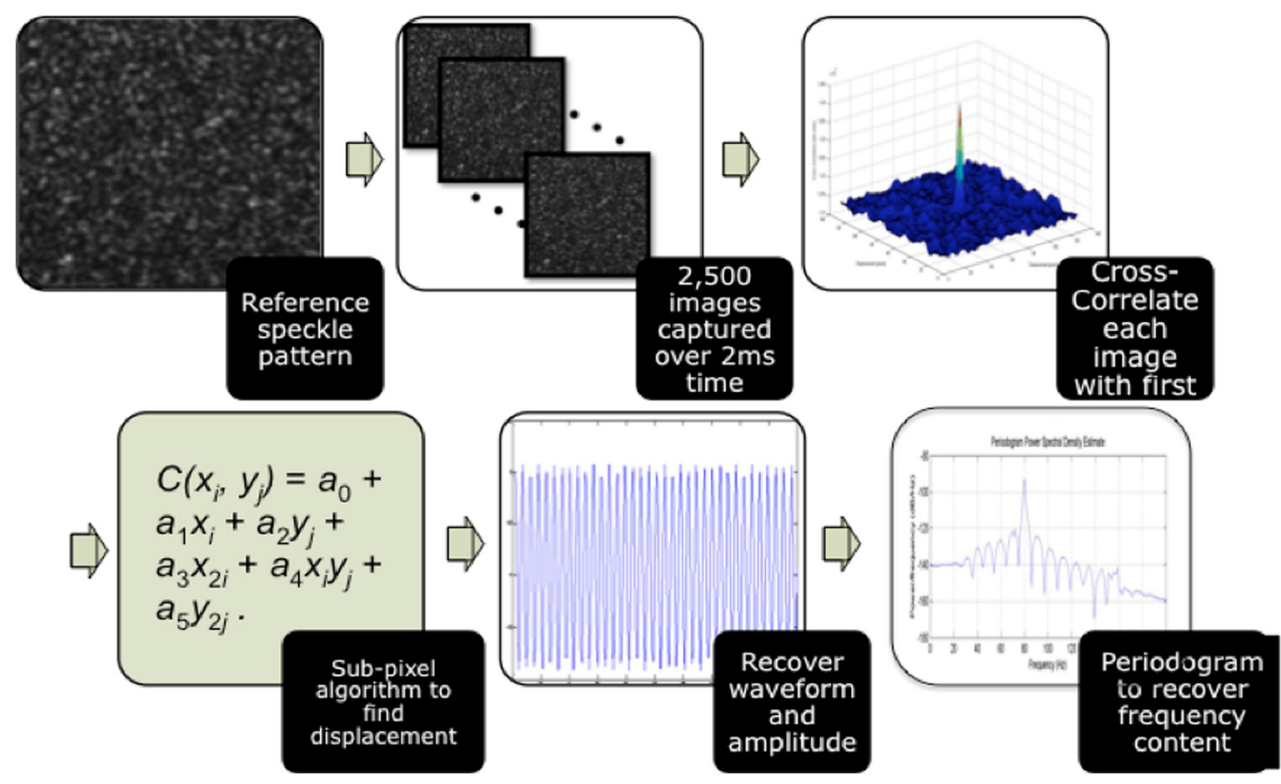

Fig. 3 Analysis procedure.
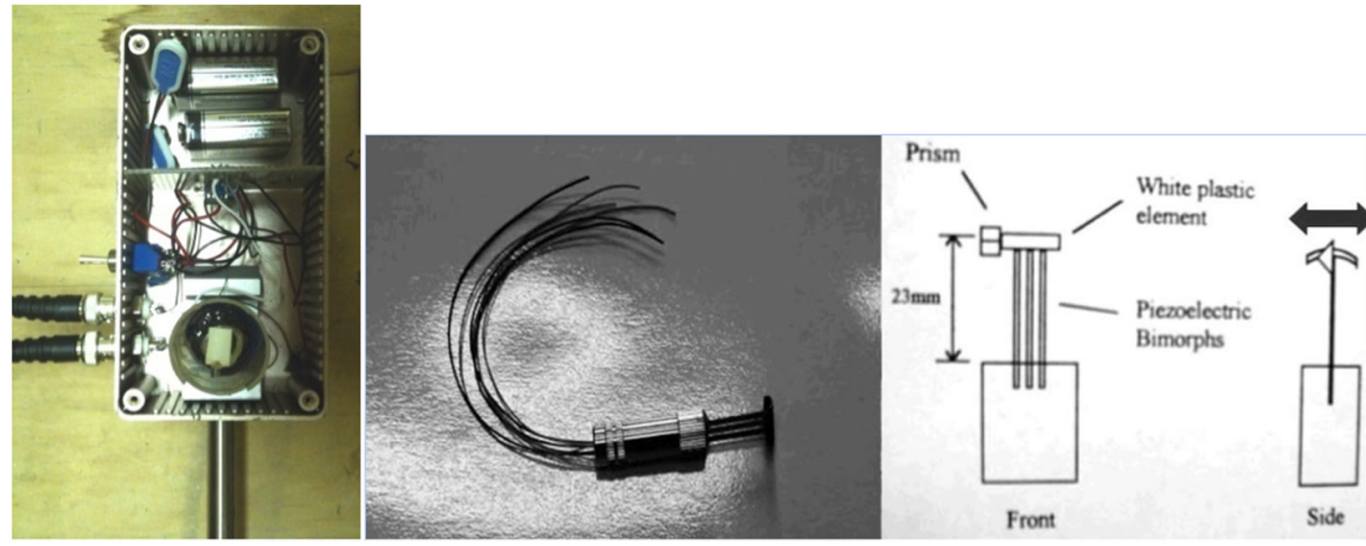

Fig. 4 OMT simulator. The small prism attached to the plastic disc is used during calibration of the simulator.

\subsubsection{Amplitude response at OMT rotation angles}

The amplitude response of the proposed setup was tested first with varying frequency and then at various input amplitudes.

To test the amplitude response with varying input amplitude, the simulator was driven at the typical mean OMT frequency of $80 \mathrm{~Hz}$ and at OMT like amplitudes ranging from 4 to $197 \mu \mathrm{rad}$ peak-peak in steps of $20 \mu \mathrm{rad}$. The experiment was repeated five times.

The lowest measurable amplitude that was recovered is given as the resolution of the system.

\subsubsection{Frequency response}

To test the frequency response, a signal generator was used to drive the simulator sinusoidally with frequencies in the OMT range ranging from 10 to $150 \mathrm{~Hz}$ in steps of $10 \mathrm{~Hz}$ at an amplitude of $197 \mu \mathrm{rad}$ peak-to-peak. $197 \mu \mathrm{rad}$ was chosen since it was the maximum amplitude achievable with the frequency generator.

\subsubsection{Robustness to setup}

Next an investigation of the impact of the $z$-axis (the optical axis) location of the target on the accuracy of the system was performed. Using a linear translation stage, the distance between the simulator and the lens was altered in steps of $1 \mathrm{~mm}$ up to a maximum allowable translation of $50 \mathrm{~mm}$. An angular displacement of $197 \mu \mathrm{rad}$ and frequency of $80 \mathrm{~Hz}$ was applied and measured at each step before the simulator was displaced again.

\subsubsection{Toleration to linear displacement during measurement}

To investigate the toleration of the system to a linear $a$-axis displacement during measurement, the translation stage was used to create a $3 \mathrm{~mm}$ displacement along the axis while the simulator was running. Experimentally, $3 \mathrm{~mm}$ was found to be the most repeatable distance at which the translation stage could be displaced by hand during the $5 \mathrm{~s}$ recording time. The experiment was performed at a typical OMT amplitude of $87 \mu \mathrm{rad}$. An $80 \mathrm{~Hz}$ sinewave signal was used to drive the simulator over a period 


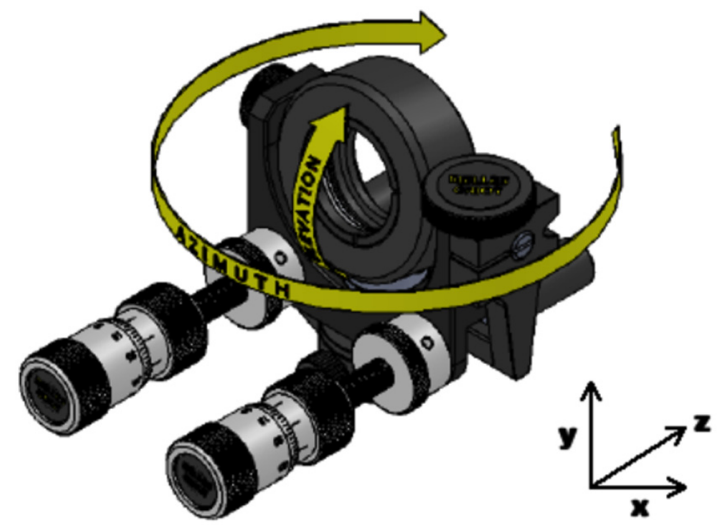

Fig. 5 Gimbal configuration.

of $5 \mathrm{~s}$ while the simulator was moved along the translation stage by hand. Each translated speckle image was correlated with the original undisplaced reference frame.

Next, the toleration of the system to an $x$-axis displacement was investigated. In this case a linear displacement of $50 \mu \mathrm{m}$ was applied by hand using the translation stage. The simulator was again set to run at $80 \mathrm{~Hz}$ and 97 urad peak-to-peak. $50 \mu \mathrm{m}$ $(4.3 \mathrm{mrad})$ was chosen since it is within the expected measurable displacement of the system $(10 \mathrm{mrad})$.

\subsection{Gimbal Tests}

A gimbal (Melles Griot, 07MAD701) was implemented to test the amplitude response of the system over a range of angles larger than those achievable with the simulator. The gimbal, shown in Fig. 5, has an azimuthal resolution of $50 \mu \mathrm{rad}$ and was used to rotate the target around the $y$-axis.

\subsubsection{Large angle amplitude response}

To test the amplitude response of the system at larger angular displacements within the dynamic range of the system, the gimbal was rotated at the following frame-to-frame angular displacements $0.2,0.25,0.5,0.75,1,2,3,4,5,6,7,8,9$, and $10 \mathrm{mrad}$. For each angle, the recorded speckle image was cross-correlated with its respective undisplaced reference image and the results were plotted.

\section{Results}

\subsection{Speckle Size}

Figure 6 shows a typical cross-correlation peak resulting from the auto-correlation of a generated speckle pattern. Assuming fully developed speckle and using Eq. (6), the size of the speckle calculated from the full width at half max of the correlation peak was found to be $33.63 \mu \mathrm{m}$. This is in close agreement with the theoretical value of $33.57 \mu \mathrm{m}$ for a beam radius of $0.6 \mathrm{~mm}$ and lens of $100 \mathrm{~mm}$ focal length. ${ }^{25}$

$$
\mathrm{FWHM}=4 \sqrt{\ln 2} \sqrt{F} \rho_{0}
$$

\subsection{Simulator Results}

\subsubsection{Amplitude response at OMT rotation angles}

Figure 7 shows the results of the amplitude response tests using the simulator. As shown in the figure, the measured angular displacements agree well with the applied angular displacement. The mean percentage error was found to be $12 \%$. At a typical OMT amplitude of $87 \mu \mathrm{rad}$ the percentage error was less than $10 \%$, below $50 \mu \mathrm{rad}$ the error increased above $10 \%$ to a maximum of $33 \%$ at $4 \mu \mathrm{rad}$.

The results show that the measurement system is capable of measuring angular displacements in the OMT range of 12 to $200 \mu \mathrm{rad}$. Further, the system resolution was determined to be at least $4 \mu \mathrm{rad}$. This is in agreement with the resolution requirements derived in Sec. 1.3.

\subsubsection{Frequency response}

The frequency response of the measured signal in the tested OMT-like range of $10-150 \mathrm{~Hz}$ was found to be flat to within $0.2 \mathrm{~dB}$, as shown in Fig. 8 .

\subsubsection{Robustness to setup}

Figure 9 shows the results of testing the response of the system to a $197 \mu \mathrm{rad}$ peak-to-peak $80 \mathrm{~Hz}$ displacement at a range of $z$-axis positions centered at $z_{1}=150 \pm 25 \mathrm{~mm}$. There is no trend in sensitivity to displacement with $z$ position. The mean measured displacement was $192 \pm 8 \mu \mathrm{rad}$. The standard

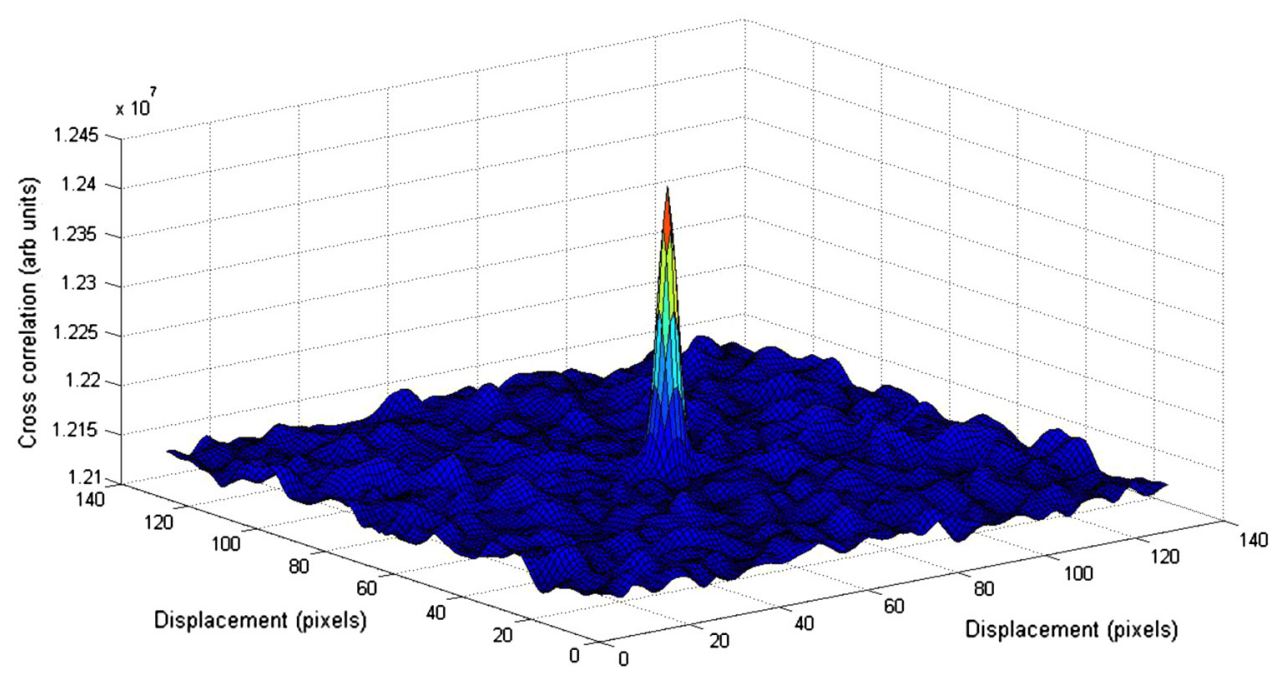

Fig. 6 Cross-correlation of speckle pattern. 


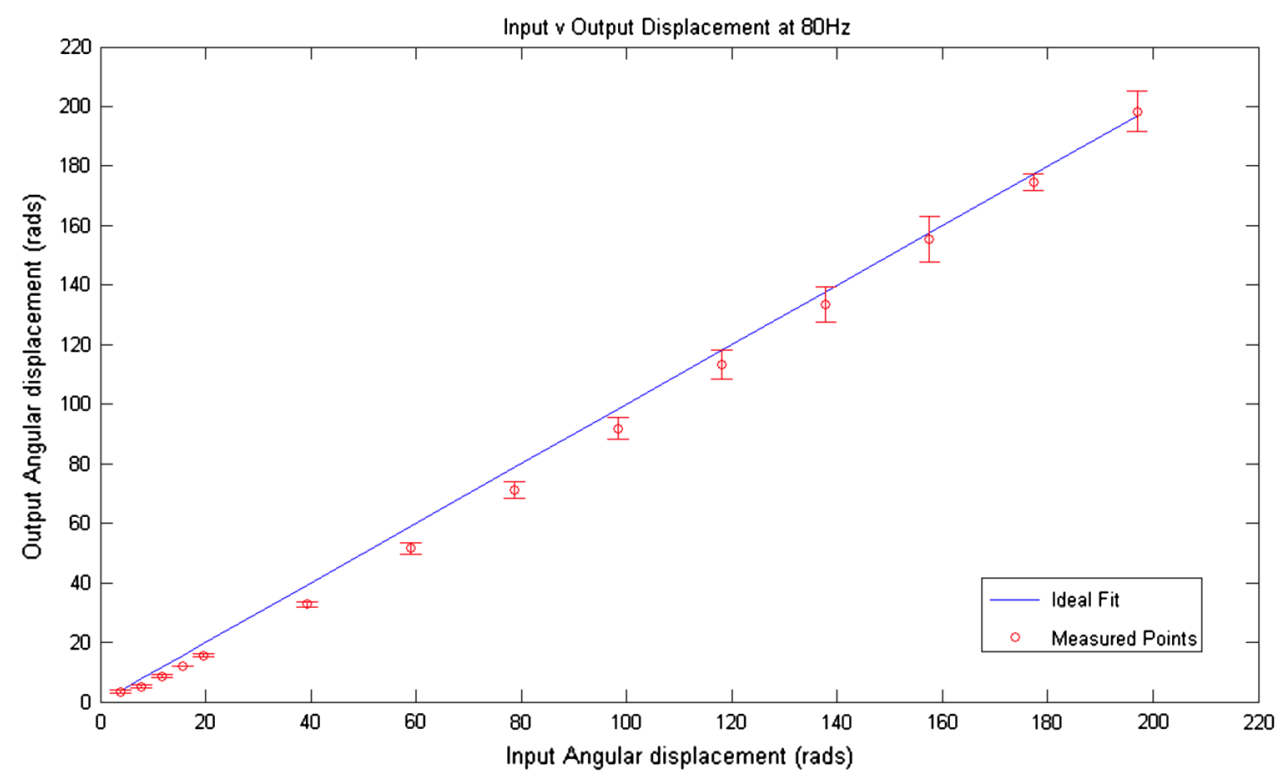

Fig. 7 Input versus output displacement for the simulator at $80 \mathrm{~Hz}$. The error bars show the standard deviation of the measurements.

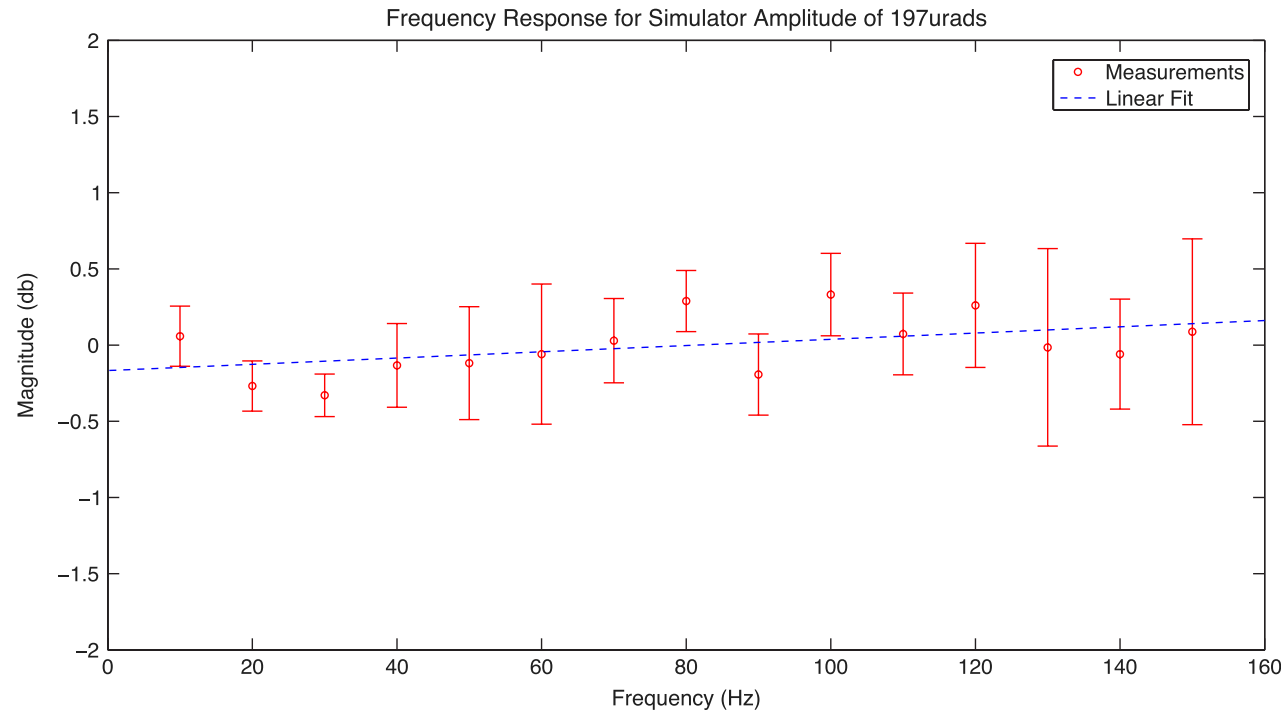

Fig. 8 Measured angle against changing simulator frequency. The error bars show the standard deviation of the measurements.

deviation with variable $z_{1}$ is similar to the standard deviation seen in Fig. 7 with repeated setup at the same $z$.

These results show no significant difference in the measured angular displacement over the $50 \mathrm{~mm}$ range in which the target distance was displaced. This result agrees with the result of Rose et al. ${ }^{25}$ The only important distance expected to contribute to the measured displacement is the lens-to-camera sensor distance. As a consequence of the invariance to target distance, all further measurements can be obtained with as short a target distance as possible in order to ensure a strong reflected light signal to the camera.

\subsubsection{Toleration to linear displacement during measurement}

The influence of a $3 \mathrm{~mm}$ linear displacement along the $z$-axis was then investigated. The approximate velocity was $600 \mu \mathrm{m} / \mathrm{s}$. As mentioned previously, the technique is expected to be invariant to movement along this axis. Figure 10 shows a plot of the unfiltered measured angular displacement for the $87 \mu \mathrm{rad}$ input. The very slight declining slope of the signal is likely to be due to a contaminating component of $x$-axis movement occurring simultaneously with the imposed $z$-axis movement.

The mean measured angular peak-to-peak displacement was found to be $77 \pm 3 \mu \mathrm{rad}$ with a resulting percentage error of $11.5 \%$. These results are comparable with the previous measurement of the angle when no extra linear displacement was enforced on the simulator. This implies that the system should be capable of tolerating at least $3 \mathrm{~mm}$ of linear $z$-axis motion. In our case, this indicates that our system should be able to accept at least $3 \mathrm{~mm}$ of linear movement from unwanted sources such as longitudinal head or body movement while still obtaining a recording of OMT.

Next, the influence of a $50 \mu \mathrm{m}$ linear displacement along the $x$-axis was investigated. As shown in Fig. 11, it was found that 


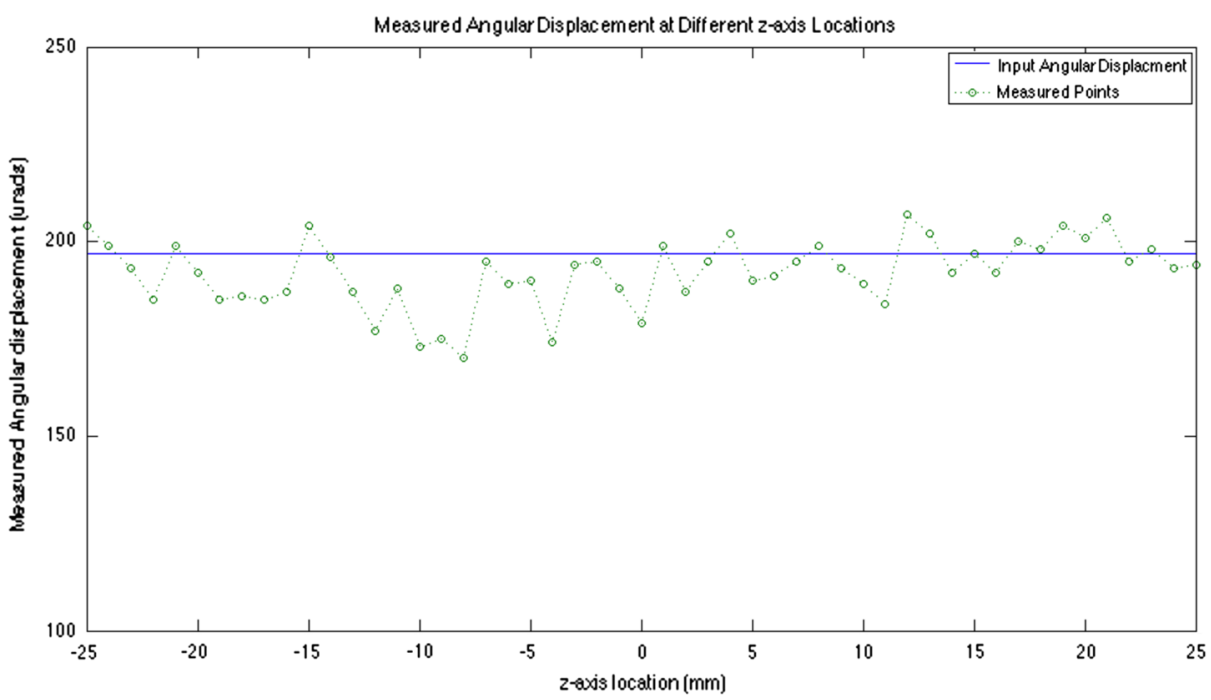

Fig. 9 Effect of linear displacement along the $z$-axis. The zero location of the $z$-axis is taken as the focal length, i.e., $z_{1}=f=150 \mathrm{~mm}$.
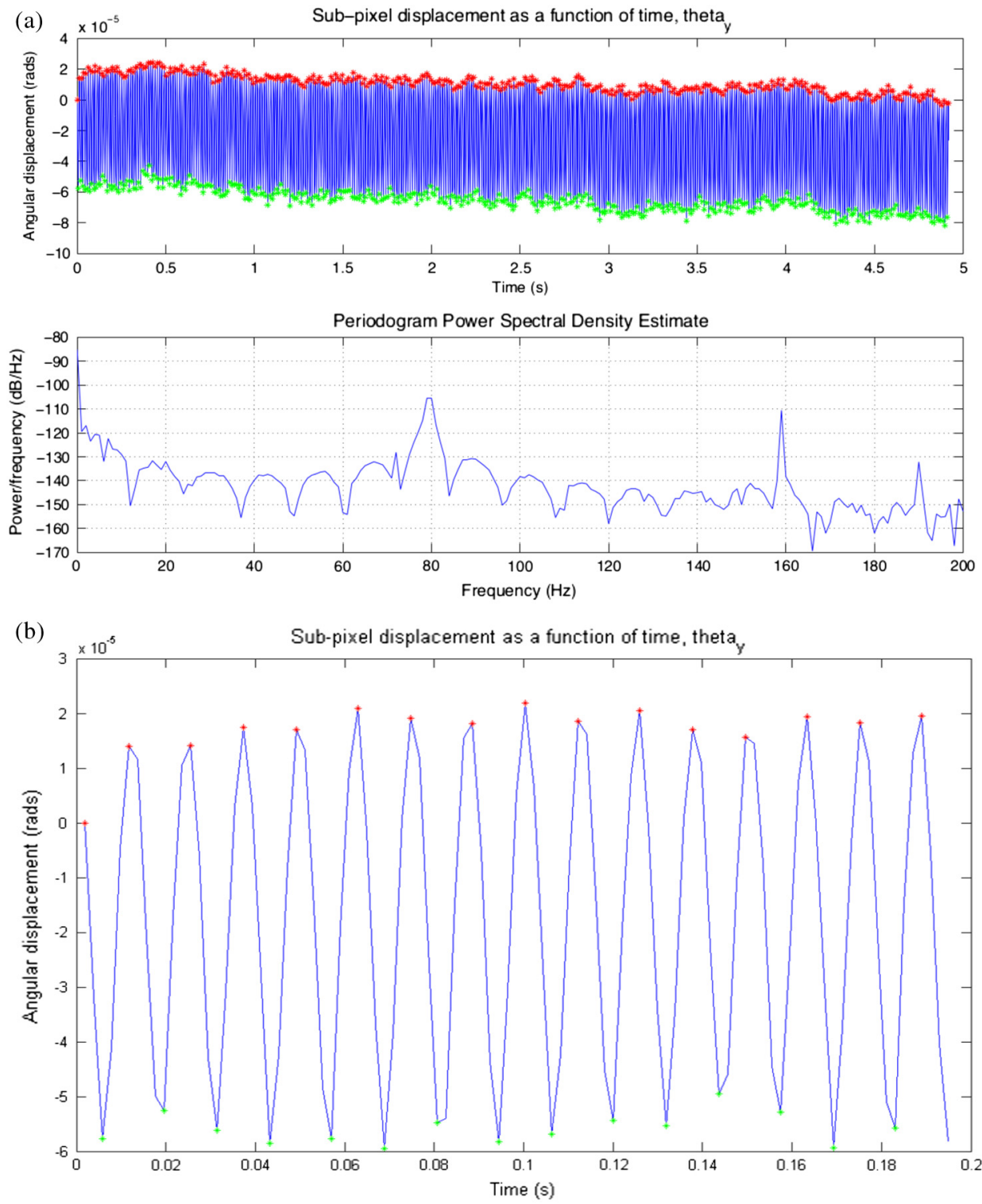

Fig. 10 (a) Measured angular displacement and periodogram for the simulator at $80 \mathrm{~Hz}, 87$ urad with an applied z-axis linear displacement. (b) Close-up version of the recovered sinewave. 
the system could recover the $87 \mu \mathrm{rad}$ input signal while the movement was imposed on it.

\subsection{Gimbal Results}

\subsubsection{Large angle amplitude response}

The results of the large angle amplitude response test using the gimbal are shown in Fig. 12. It was found that once a frame-toframe displacement of $6 \mathrm{mrad}$ was reached, the system could not provide a reliable measurement of the angular displacement. This is likely to be a result of a decorrelation between the speckle pattern taken after $5 \mathrm{mrad}$ and that of the reference speckle pattern. The system could not measure a frame-toframe displacement of $10 \mathrm{mrad}$, the theoretical maximum frame-to-frame measurable angular displacement of the system, if decorrelation is ignored.

In an attempt to overcome this difficulty, the crosscorrelation algorithm was altered so that each speckle image was cross-correlated with the preceding image. Using this altered
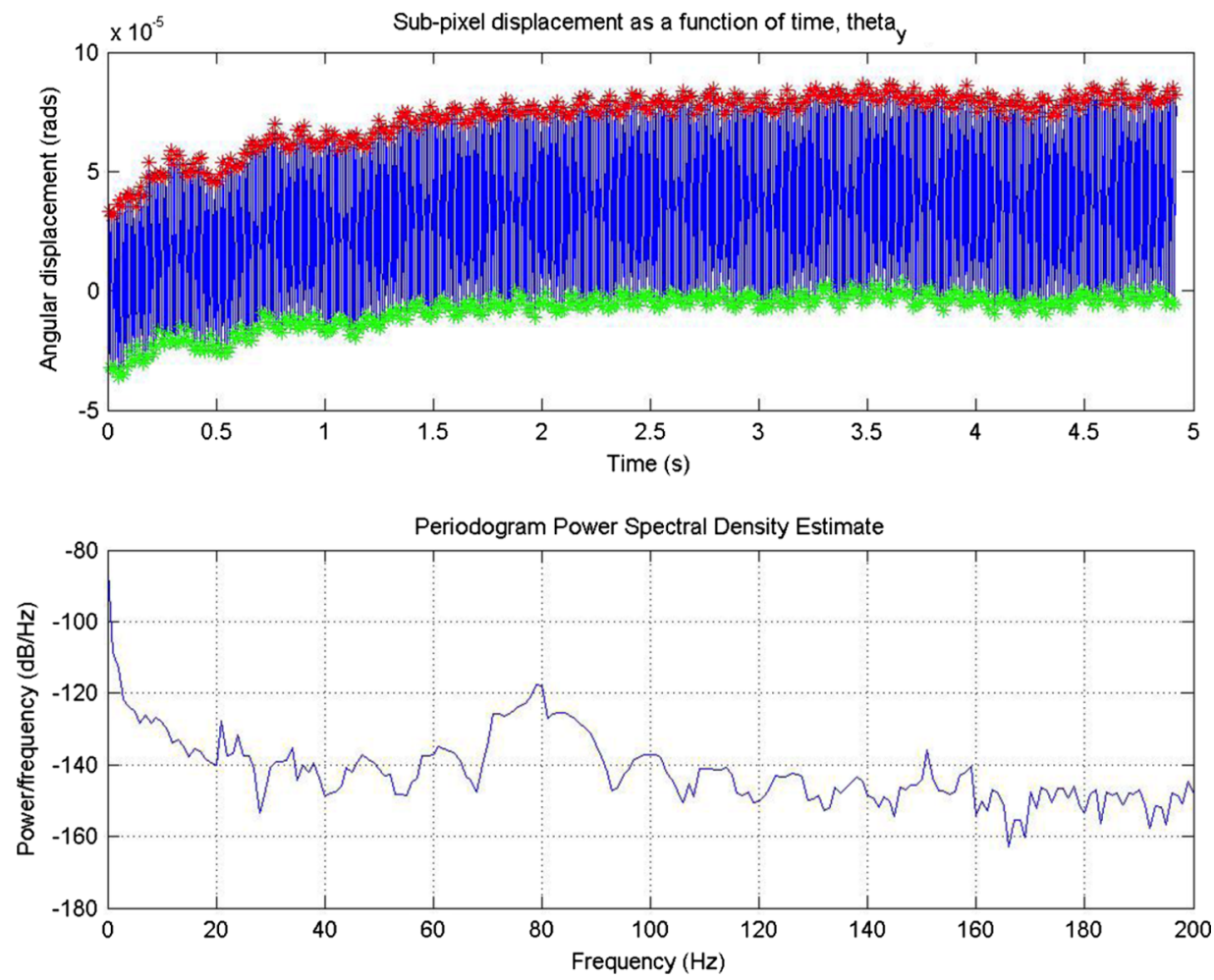

Fig. 11 Measured angular displacement and periodogram for the simulator at $80 \mathrm{~Hz}, 87$ urad with an applied $x$-axis linear displacement.

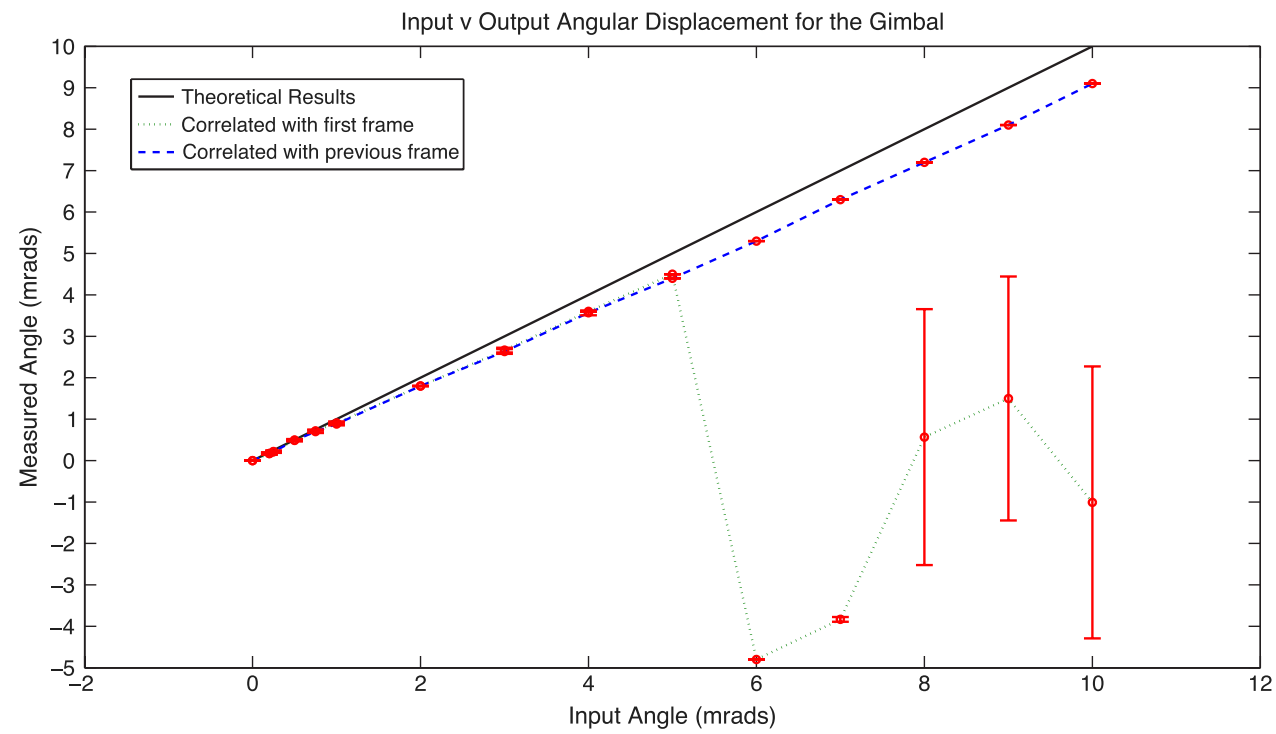

Fig. 12 Applied $v$ measured angular displacement of the gimbal in steps of $1 \mathrm{mrad}$. The dotted line shows the results from correlating each speckle frame with the original undisplaced reference frame. The dashed line shows the result when each frame is correlated with the previous frame. 


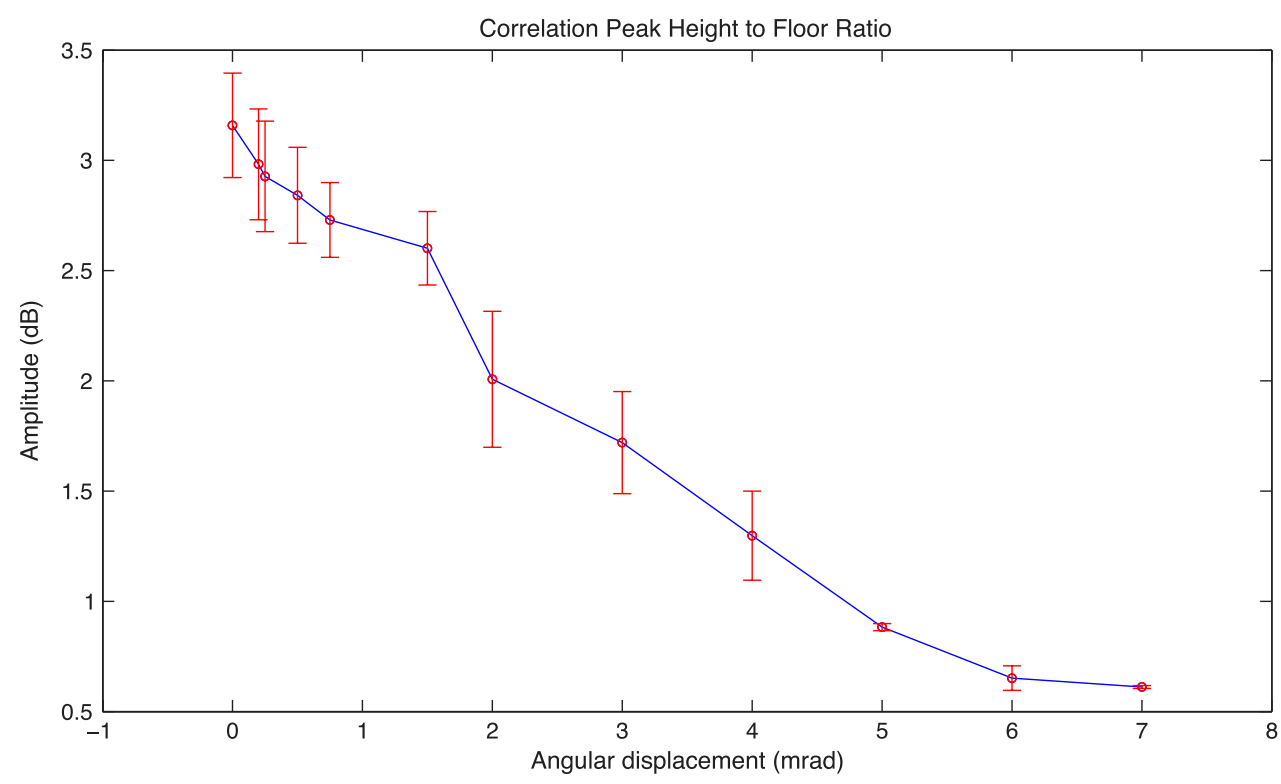

Fig. 13 Decreasing correlation peak height to mean floor ratio for increasing frame-to-frame displacement.

algorithm, the system was capable of measuring up to $10 \mathrm{mrad}$. The mean percentage error on these results was $10.5 \pm 3 \%$ over the range of measurements with a maximum error of $15 \%$.

Both methods of measuring the output angular displacement are compared in Fig. 12. The measurement results agree well with the input, although the error begins to increase to approximately $10 \%$ at $1 \mathrm{mrad}$. As outlined in Sec. 1.1.3, a microsaccade is expected to create a frame-to-frame displacement of $1.3 \mathrm{mrad}$. These results show that the system will be able to measure such a displacement, although the measured amplitude may be lower than expected.

As an evaluation of the decorrelation between the images, the cross-correlation peak height for image frames correlated with the undisplaced reference frame was measured. The value of the peak height was then divided by the mean floor value to calculate a ratio that could be compared across different image sets. As shown in Fig. 13, the peak height to floor ratio decreased with increasing frame-to-frame displacement. This is to be expected since the frames were becoming progressively more different.

\section{Discussion}

Overall the system was found to be capable of measuring frequencies and angular displacements within the OMT range of 12 to $200 \mu \mathrm{rad}$. Experimentally, the resolution was found to be $4 \mu \mathrm{rad}$ as expected. This is close to the design requirement for an OMT measurement system.

The amplitude response of the system was found to be insensitive to target locations of up to $\pm 25 \mathrm{~mm}$ along the optical axis. This has important outcomes in vivo as it means there is no strict target distance requirements. It will help ease the setup alignment since it is expected that there would be a variance in the target distance as each person places his head in the headrest at slightly different locations.

A linear displacement of the target during measurement of an OMT-like angular rotation at $80 \mathrm{~Hz}$ along the optical axis of up to $3 \mathrm{~mm}$ was found not to influence the measurement results. This insensitivity is important as it implies that angular displacements of the sclera can still be measured even if the head moves linearly during the recording.

Angular displacements larger than those of OMT were also investigated. It was found that the system was capable of measuring frame-to-frame angular displacements in the order of microsaccades $(1.3 \mathrm{mrad})$ and in the order of the total expected displacement frame-to-frame displacement from all eye and head movements $(1.56 \mathrm{mrad})$.

The accuracy of the frame-to-frame displacement began to diminish at $6 \mathrm{mrad}$. The maximum expected frame-to-frame displacement is $1.56 \mathrm{mrad} .5 \mathrm{mrad}$ sets a limit as to how much displacement can be tolerated relative to a reference frame. As discussed previously, the maximum expected angular displacement frame-to-frame for a handheld device would be $2.84 \mathrm{mrad}$; this falls within our measurable range, and so the speckle correlation system could theoretically be suitable for such a handheld device.

\section{Conclusions}

A novel noncontact method for measuring OMT was reviewed. The parameters required for accurate measurement and methods to achieve them by implementing a speckle-based measurement of angular rotations were discussed. A resolution of $4 \mu \mathrm{rad}$ and dynamic range of 4 to $5000 \mu \mathrm{rad}$ was achieved with the system. It was shown that decorrelation due to other head/body movements will not be large enough to have a detrimental effect on OMT measurement, and that its signal should be recovered from a background of these movements.

Future work should include the effects of measuring speckle from living objects since this would involve a secondary type of boiling speckle known as biospeckle. ${ }^{33-36}$ Biospeckle will cause the speckle patterns to change in time. It is expected that this random time varying speckle will occur together with the wanted translational speckle from OMT movement. Biospeckle will decorrelate the speckle images and be a source of noise in the images. The rates of biospeckle from the human eye sclera have never been quantified nor has their influence on eye movement ever been investigated. To separate OMT-related speckle pattern movements from biospeckle movements, the properties 
of in vivo biospeckle from the sclera will need to be investigated. Further optimization of the proposed optical system may be required to overcome this biospeckle obstacle.

\section{Acknowledgments}

This work is based on research supported by Science Foundation Ireland.

\section{References}

1. F. H. Adler and M. Fliegelman, "Influence of fixation on the visual acuity," Arch. Ophthal. 12(4), 475-483 (1934).

2. S. Martinez-Conde, S. L. Macknik, and D. H. Hubel, "The role of fixational eye movements in visual perception," Nat. Rev. Neurosci. 5(3), 229-240 (2004).

3. C. Bolger et al., "Dominant frequency content of ocular microtremor from normal subjects," Vis. Res. 39(11), 1911-1915 (1999).

4. C. Bolger et al., "High-frequency eye tremor reliability of measurement," Clin. Phys. Physiol. Meas. 13(2), 151-159 (1992).

5. D. Coakley and J. G. Thomas, "Ocular microtremor: aneurogenic phenomenon," Electroencephalogr. Clin. Neurophysiol. 19(4), 325328 (1979).

6. S. Bojanic and C. Bolger, "Ocular microtremor (OMT): a useful indicator of outcome in coma?" Br. J. Anaesth. 82(5), 795P-796P (1999).

7. C. Bolger et al., "Ocular microtremor (OMT): a new neurophysiological approach to multiple sclerosis," J. Neurol. Neurosurg. Psychiatr. 68(5), 639-642 (2000).

8. C. Bolger et al., "Ocular microtremor in patients with idiopathic Parkinson's disease," J. Neurol. Neurosurg. Psychiatr. 66(4), 528531 (1999).

9. D. Coakley, J. G. Thomas, and J. N. Lunn, "Effect of anesthesia on ocular microtremor,"Br. J. Anaesth. 48(11), 1122-1123 (1976).

10. M. Heaney et al., "Ocular microtremor during general anesthesia: results of a multicenter trial using automated signal analysis," Anesthesia and Analgesia 99(3), 775-780 (2004).

11. N. Collins, "Ocular microtremor as a clinical and scientific tool in neurologic disease; validation and application of a generalised discovery protocol," Ph.D. Thesis, University of Dublin, Trinity College (2011).

12. A. Spauschus et al., "The origin of ocular microtremor in man," Exp. Brain Res. 126(4), 556-562 (1999).

13. C. Bolger et al., "Ocular microtremor in oculomotor palsy," J. Neuro. Ophthalmol. 19(1), 42-45 (1999).

14. H. Bengi and J. G. Thomas, "3 Electronic methods for recording ocular tremor," Med. Biol. Eng. 6(2), 171-179 (1968).

15. N. Sheahan et al., "Ocular microtremor measurement system: design and performance," Med. Biol. Eng. Comput. 31(3), 205-212 (1993).
16. N. Sheahan, "Ocular microtremor measurement technique and biophysical analysis," Ph.D. Thesis, Trinity College Dublin (1991).

17. N. F. Sheahan et al., "Sources ofvariance inocularmicrotremor," Physiol. Meas. 15(1), 101-106 (1994).

18. G. Boyle, D. Coakley, and J. F. Malone, "Interferometry for ocular microtremor measurement," Appl. Opt. 40(1), 167-175 (2001).

19. J. P. Ryle et al., "Compact portable ocular microtremor sensor: design, development and calibration," J. Biomed. Optic. 14(1), 014021 (2009),

20. J. P. H. Reulen et al., "Precise recording of eye-movement: the IRIS technique 1," Med. Biol. Eng. Comput. 26(1), 20-26 (1988).

21. D. A. Robinson, "A method of measuring eye movement using a scleral search coil in a magnetic field,"IEEE Trans. Bio. Eng. BM10(4), 137145 (1963).

22. M. Al-Kalbani, "Ocular microtremor measurement, characterization \& analysis," Ph.D. Thesis, Trinity College, University of Dublin (2009).

23. H. T. Kasprzak and D. R. Iskander, "Ultrasonic measurement of fine head movements in a standard ophthalmic headrest," IEEE Trans. Instrum. Meas. 59(1), 164-170 (2010).

24. R. Greenwood, Neurological Rehabilitation, p. 173, Psychology Press, Bath (1997).

25. B. Rose, H. Imam, and S. G. Hanson, "Non-contact laser speckle sensor for measuring one- and two-dimensional angular displacement,"J. Opt. 29(3), 115-120 (1998).

26. B. Rose et al., "Laser-speckle angular-displacement sensor: theoretical and experimental study," Appl. Opt. 37(11), 2119-2129 (1998).

27. IEC, "Safety of laser products," Vol. IEC 60825-1 ed2.0, International Electrotechnical Commission, Geneva (2007).

28. ANSI, "American National Standard for Safe Use of Lasers," Vol. ANSI Z136.1, ed American National Standards Institution (2007).

29. ANSI, "Safe Use of Lasers," Vol. ANSI Z136.1-2007, American National Standards Institute (2007).

30. Photometrics, Photometrics, Arizona, http://www.photometrics.com (2010).

31. Matlab Version R2009a, The MathWorks Inc., Natick, Massachusetts (2010).

32. P.-C. Hung and A. S. Voloshin et al., "In-plane strain measurement by digital image correlation," J. Braz. Soc. Mech. Sci. Eng. 25, 215-221 (2003).

33. H. J. Rabal and R. A. Braga, Dynamic Laser Speckle and Applications, CRC Press, Florida (2008).

34. R. A. Braga et al., "Reliability of biospeckle image analysis," Optic. Laser. Eng. 45(3), 390-395 (2007).

35. A. Oulamara, G. Tribillon, and J. Duvernoy, "Biological-activity measurement on botanical specimen surfaces using a temporal decorrelation effect of laser speckle," J. Mod. Optic. 36(2), 165-179 (1989).

36. Z. Xu, C. Joenathan, and B. M. Khorana, "Temporal and spatial properties of the time-varying speckles of botanical specimens,"Opt. Eng. 34(5), 1487-1502 (1995). 